\title{
Identification of Ulceration in Early Gastric Cancer before Resection is Not Easy: Need for a New Guideline for Endoscopic Submucosal Dissection Indication Based on Endoscopic Image
}

\author{
Hang Lak Lee \\ Department of Gastroenterology, Hanyang University Hospital, Seoul, Korea
}

See "Can Endoscopic Ulcerations in Early Gastric Cancer Be Clearly Defined before Endoscopic Resection? A Survey among Endoscopists" by Sung Min Park, Byung-Wook Kim, Joon Sung Kim, et al., on page 473-478.

Endoscopic submucosal dissection (ESD) is a powerful method for treating gastric epithelial neoplasms. The presence of ulceration is one of the most important factors that determine the possibility for ESD treatment in patients with early gastric cancer (EGC). Guidelines for gastric cancer suggest that four factors should be defined to choose endoscopic resection as the treatment modality: size, invasion depth, pathological differentiation, and presence or absence of ulceration. ${ }^{1}$ For the endoscopist, the presence of ulceration in EGC should be determined before ESD. Therefore, ulceration in EGC should be determined on the basis of the endoscopic findings in clinical practice. Sometimes, misinterpretation of endoscopic ulcerations can result in a more aggressive treatment; therefore, exact determination of ulceration is important in selecting either endoscopic resection or surgery. About 20 years ago, Gotoda et al. suggested the indication of endoscopic resection after review of surgical specimens. ${ }^{2}$ In this study, ulceration, or ulcer scar, was defined as converging folds, deformity of the muscularis propria, or fibrosis in the submucosa or the deeper layers, without considering endoscopic image

Received: September 3, 2017 Accepted: September 22, 2017

Correspondence: Hang Lak Lee

Department of Gastroenterology, Hanyang University Hospital, 222-1 Wangsimni-ro Seongdong-gu, Seoul 04763, Korea

Tel: +82-02-2290-8354, Fax: +82-02-2298-9183, E-mail: alwayshang@hanyang.ac.kr

(c) This is an Open Access article distributed under the terms of the Creative Commons Attribution Non-Commercial License (http://creativecommons.org/ licenses/by-nc/3.0) which permits unrestricted non-commercial use, distribution, and reproduction in any medium, provided the original work is properly cited. findings.

In this issue of Clinical Endoscopy, Park et al. performed a questionnaire survey composed of a summary of each case and endoscopic images of 7 patients with EGC. ${ }^{3}$ This study suggested that the duration of endoscopic practice (more than 5 years) and experience with ESD were the significant factors associated with accurate endoscopic diagnosis of ulcer. However, although the duration of endoscopic practice and experience with ESD were the significant factors, even for experienced endoscopists, diagnostic accuracy was approximately $28 \%$ in the case of image C. In this paper, image C showed a shallow mucosal break without converging folds. The authors also commented that endoscopic ulcerations in EGC are quite difficult to define; therefore, this overestimation may result in more aggressive treatment. We also agree with this comment and performed one unpublished study about the endoscopic diagnosis of ulceration in EGC. We analyzed the endoscopic and pathological findings from 104 patients who underwent ESD or curative gastrectomy for EGC that showed endoscopic ulcers. Two expert pathologists reviewed the pathological slides again. The discrepancy between the endoscopic and pathological ulcers was 46.15\% (48/104). The 48 cases that showed discrepancy were EGC IIc type lesions, with only erosive lesions on the pathological review. Our study also demonstrated the difficulty of endoscopic diagnosis of ulceration in EGC.

The main limitation of ESD is the presence of micrometastasis. ${ }^{4-8}$ Although curative surgical resection was performed 
in EGC, the recurrence rate was approximately $1.7 \%-3.4 \%$, which may be due to micrometastasis. According to a previous report, tumor size, macroscopic type, accompanying ulcers, and depth of invasion are strongly associated with micrometastasis. ${ }^{9}$ Therefore, tumors with suspected accompanying ulcers may have a recurrence risk due to micrometastasis, which may indicate the inappropriateness of ESD. For these reasons, therapeutic endoscopists can hesitate to perform ESD in such cases, especially in those with accompanying ulcers.

Gotoda et al. $^{2}$ suggested the indication of endoscopic resection for EGC after investigating surgical specimens about 20 years ago without consideration of the endoscopic finding. Until now, this indication has been accepted as a universal truth among therapeutic endoscopists. However, endoscopic optical technology has been rapidly improving during the recent 20 years; therefore, the resolutions provided by the recent endoscopic imaging modalities cannot compare with those provided by preceding endoscopic imaging modalities. In addition, large ESD data have been accumulated since the introduction of the ESD procedure. A new guideline of ESD indication should be introduced mainly on the basis of endoscopic imaging findings for more accurate patient selection for ESD. Considering the high discordance rate of ulceration between endoscopic and pathological findings, performing an endoscopic resection for a confirmative diagnosis before gastrectomy can be a good option.

In conclusion, even for experienced endoscopists, accurate diagnosis of ulceration in EGC is not easy. Overestimation may result in a more aggressive treatment, so careful endo- scopic inspection and minute observation are needed when considering the ESD procedure. We look forward to a new ESD guideline that is easy to use by endoscopists.

\section{Conflicts of Interest}

The author has no financial conflicts of interest.

\section{REFERENCES}

1. Japanese Gastric Cancer Association. Japanese gastric cancer treatment guidelines 2010 (ver. 3). Gastric Cancer 2011;14:113-123.

2. Gotoda T, Yanagisawa A, Sasako M, et al. Incidence of lymph node metastasis from early gastric cancer: estimation with a large number of cases at two large centers. Gastric Cancer 2000;3:219-225.

3. Park SM, Kim BW, Kim JS, Kim YW, Kim GJ, Ryu SJ. Can endoscopic ulcerations in early gastric cancer be clearly defined before endoscopic resection? A survey among endoscopists. Clin Endosc 2017;50:473-478.

4. Cai J, Ikeguchi M, Maeta M, Kaibara N. Micrometastasis in lymph nodes and microinvasion of the muscularis propria in primary lesions of submucosal gastric cancer. Surgery 2000;127:32-39.

5. Nakajo A, Natsugoe S, Ishigami S, et al. Detection and prediction of micrometastasis in the lymph nodes of patients with pN0 gastric cancer. Ann Surg Oncol 2001;8:158-162.

6. Maehara Y, Oshiro T, Endo K, et al. Clinical significance of occult micrometastasis lymph nodes from patients with early gastric cancer who died of recurrence. Surgery 1996;119:397-402.

7. Harrison LE, Choe JK, Goldstein M, Meridian A, Kim SH, Clarke K. Prognostic significance of immunohistochemical micrometastases in node negative gastric cancer patients. J Surg Oncol 2000;73:153-157.

8. Cai J, Ikeguchi M, Tsujitani S, Maeta M, Liu J, Kaibara N. Significant correlation between micrometastasis in the lymph nodes and reduced expression of E-cadherin in early gastric cancer. Gastric Cancer 2001;4:66-74.

9. Gotoda T. Endoscopic resection of early gastric cancer. Gastric Cancer 2007;10:1-11. 